\title{
Insulin Sensitivity and Secretion in Arab Americans with Glucose Intolerance
}

\author{
Francine D. Salinitri, PharmD, ${ }^{1,2}$ Nicole R. Pinelli, PharmD, MS, CDE, \\ Emily T. Martin, PhD, and Linda A. Jaber, PharmD ${ }^{1}$
}

\begin{abstract}
Background: This study examined the pathophysiological abnormalities in Arab Americans with impaired fasting glucose (IFG) and/or impaired glucose tolerance (IGT).

Subjects and Methods: Homeostasis model assessment of insulin resistance (HOMA-IR), homeostasis model assessment of

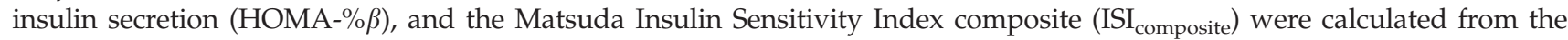
fasting and stimulated glucose and insulin concentrations measured during the oral glucose tolerance test in a populationbased, representative, cross-sectional sample of randomly selected Arab Americans.

Results: In total, 497 individuals (42 \pm 14 years old; 40\% males; body mass index [BMI], $29 \pm 6 \mathrm{~kg} / \mathrm{m}^{2}$ ) were studied. Multi-

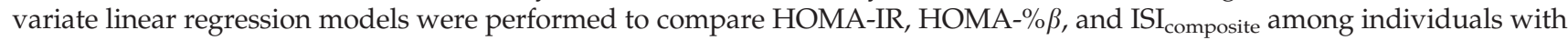
normal glucose tolerance (NGT) $(n=191)$ versus isolated IFG $(n=136)$, isolated IGT $(n=22)$, combined IFG/IGT $(n=43)$, and diabetes $(n=105)$. Compared with individuals with NGT $(2.9 \pm 1.6)$, HOMA-IR progressively increased in individuals with isolated IFG $(4.8 \pm 2.7, P<0.001)$, combined IFG/IGT $(6.0 \pm 4.3, P<0.001)$, and diabetes $(9.7 \pm 8.3, P<0.001)$ but not in those with isolated IGT $(3.0 \pm 1.7, P=0.87)$. After adjustment for sex and BMI, these associations remained unchanged. Whole-body insulin sensitivity as measured by ISI $_{\text {composite }}$ was significantly lower in individuals with isolated IFG $(3.9 \pm 2.3, P<0.001)$, isolated IGT $(2.8 \pm 1.5, P<0.001)$, combined IFG/IGT $(1.9 \pm 1.1, P<0.001)$, and diabetes $(1.6 \pm 1.1, P<0.001)$ compared with those with NGT $(6.1 \pm 3.5)$. HOMA- $\% \beta$ was significantly lower in diabetes $(113.7 \pm 124.9, P<0.001)$ compared with NGT

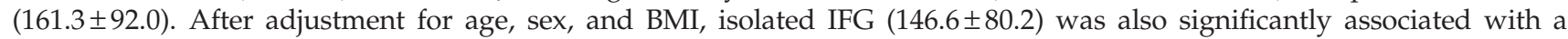
decline in HOMA- $\% \beta$ relative to NGT $(P=0.005)$.

Conclusions: This study suggests that differences in the underlying metabolic defects leading to diabetes in Arab Americans with IFG and/or IGT exist and may require different strategies for the prevention of diabetes.
\end{abstract}

\section{Introduction}

TMPAiRED FASTing GLUCOSE (IFG) and impaired glucose tolerance (IGT) have been identified as independent precursors for the development of type 2 diabetes. ${ }^{1-3}$ Studies have illustrated that different pathophysiological abnormalities characterize isolated IFG and isolated IGT. Individuals with isolated IFG have decreased hepatic insulin sensitivity accompanied by reduction in basal and first-phase insulin secretion. ${ }^{3-6}$ Those with isolated IGT have minimal reduction in hepatic insulin sensitivity but instead have predominant reduction in peripheral insulin sensitivity and reduced firstand second-phase insulin secretion..$^{3-5,7,8}$ In patients with combined IFG/IGT, decreased hepatic/peripheral insulin sensitivity and profound reduction in insulin secretion have been documented. ${ }^{3,4,6}$

Emerging evidence also suggests that ethnic variation in the natural history, etiology, and underlying pathology of IFG and IGT exists. ${ }^{4,8-10}$ Abdul-Ghani et al. ${ }^{10}$ demonstrated differences in the degree of insulin secretion and insulin sensitivity among different ethnic populations with isolated IGT; specifically, Arabs had a significantly greater reduction in insulin secretion and lower reduction in insulin sensitivity compared with Japanese and Mexican Americans. Decreased insulin sensitivity was the predominant contributory factor to the decline in $\beta$-cell function in Mexican Americans, whereas

\footnotetext{
${ }^{1}$ Department of Pharmacy Practice, Wayne State University Eugene Applebaum College of Pharmacy and Health Sciences, Detroit, Michigan.

${ }^{2}$ Department of Pharmacy Services, Oakwood Hospital and Medical Center, Dearborn, Michigan

${ }^{3}$ University of North Carolina Eshelman School of Pharmacy, Chapel Hill, North Carolina.

${ }^{4}$ Department of Pharmacy, University of North Carolina Hospitals and Clinics, Chapel Hill, North Carolina.

An abstract describing parts of this study was presented at the $72^{\text {nd }}$ American Diabetes Association Scientific Sessions, Philadelphia, PA, June 10, 2012.
} 
the decline in both insulin sensitivity and secretion influenced conversion from normal glucose tolerance (NGT) to IGT in Japanese subjects. ${ }^{10}$ In another study, a more significant impairment in $\beta$-cell compensation for the degree of insulin sensitivity was observed in Arab women with gestational diabetes compared with Scandinavian women after adjustment for body mass index (BMI). ${ }^{11}$

We have demonstrated that the prevalence of diabetes and prediabetes is disproportionately high among Arab Americans, posing a major public health burden. ${ }^{12}$ Greater insight into the pathophysiological abnormalities leading to diabetes in this population will facilitate the identification of pharmacological strategies that may be used as early interventions to prevent or delay the disease progression. ${ }^{13}$ Insulin sensitivity and $\beta$-cell function have been examined in a small number of Arabs with isolated IGT, ${ }^{10}$ but to date no studies have described the transition in glucose homeostasis from NGT to IFG, IGT, or combined IFG/IGT in this population. In this analysis, we aim to compare the pathophysiological defects of isolated IFG, isolated IGT, combined IFG/IGT, and diabetes relative to NGT in a representative, cross-sectional sample of Arab Americans. Preliminary results have been presented elsewhere in abstract form. ${ }^{14}$

\section{Subjects and Methods}

The methods of this cross-sectional, population-based study have been described in detail elsewhere. ${ }^{12}$ In brief, an initial sampling frame of households was constructed in two geographical areas of Dearborn, MI, that are predominantly inhabited by Arab Americans. Nonpregnant adults 20-75 years of age with native Arab ancestry were randomly selected from these sampling frames. Arab Americans were defined by self-report of Arab ancestry of the individual, a parent, or a grandparent. The study was approved by the Wayne State University Institutional Review Board. All participants provided written informed consent.

Eligible individuals reported to the clinic following a 12-h overnight fast. Standardized questionnaires translated into Arabic were used to assess demographic factors. Height and body weight were measured in light clothing and without shoes. BMI was calculated as the body weight (in kilograms) divided by the square of the height (in meters).

Fasting blood samples were collected for measurement of plasma glucose and serum insulin and lipid concentrations. Individuals without documented diabetes underwent a 75-g oral glucose tolerance test (OGTT), and blood samples were collected at $120 \mathrm{~min}$ for measurement of plasma glucose and serum insulin levels. Plasma glucose concentrations were measured by an automated glucose oxidase method using Behring Diagnostics (La Jolla, CA) reagents (SVR glucose test). The serum insulin level was measured with a double antibody radioimmunoassay (Linco Research, St. Charles, $\mathrm{MO})$ and was standardized against the International Reference Preparation (National Institute for Biological Standards and Control, Potters Bar, Hertfordshire, United Kingdom). Total cholesterol and triglyceride concentrations were measured using enzymatic colorimetric techniques (Cobas Mira Chemstation; Roche, Indianapolis, IN). Highdensity lipoprotein cholesterol was measured with a highdensity lipoprotein direct assay using the elimination approach and meeting the National Cholesterol Education
Program guidelines for precision and accuracy (Cobas Mira Chemstation). Low-density lipoprotein cholesterol was calculated using the equation of Friedewald et al. ${ }^{15}$

Individuals were considered to have diabetes if they reported a previous medical diagnosis of diabetes and/or were using insulin or oral antihyperglycemic agents. Glucose tolerance status of individuals without a previous diagnosis of diabetes was defined according to the 2012 American Diabetes Association OGTT-based diagnostic criteria. ${ }^{16}$

Insulin resistance was estimated using the homeostasis model assessment (HOMA) of insulin resistance (HOMA-IR) and was defined as (fasting serum insulin [in $\mu \mathrm{U} / \mathrm{mL}] \times$ fasting plasma glucose [in mmol/L])/22.5. ${ }^{17}$ Insulin secretion was calculated using the homeostasis model assessment of $\beta$ cell function (HOMA- $\% \beta$ ) and was calculated as (fasting serum insulin [in $\mu \mathrm{U} / \mathrm{mL}] \times 20)] /(\mathrm{FPG}[$ in $\mathrm{mmol} / \mathrm{L}]-3.5) .{ }^{17}$

Insulin sensitivity was also measured using the Matsuda

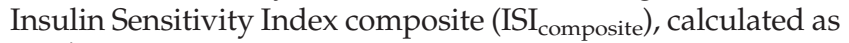
$\left[k / \sqrt{ }\left(G_{0} \times I_{0} \times G_{120} \times I_{120}\right)\right]$, where $k(=10,000)$ is the constant, $G_{0}$ and $G_{120}$ are the plasma glucose concentrations at times 0 and $120 \mathrm{~min}$, respectively, and $I_{0}$ and $I_{120}$ are the plasma insulin concentrations at times 0 and $120 \mathrm{~min}$, respectively. ${ }^{18}$

Descriptive statistics were performed to compare the demographic characteristics of participants by glucose tolerance status. Data are expressed as mean $\pm S D$ values or percentages. Depending on whether the outcome measure was continuous or categorical, data were analyzed using analysis of variance or $\chi^{2}$ test, respectively. Multivariate linear regression models for HOMA-IR, HOMA- $\% \beta$, and ISI $_{\text {composite }}$ were built with backward selection with a removal threshold of $P=0.2$ with linear age, linear BMI, and sex considered as covariates. Analyses were completed using the statistical software package STATA version 11.0 (StataCorp, College Station, TX). A two-tailed $P<0.05$ was considered statistically significant.

\section{Results}

In total, 542 Arab Americans participated in this study. Of these, 45 individuals without a history of diabetes did not have complete OGTT data and were excluded from this analysis, for a final study sample of 497. Demographic characteristics are presented in Table 1 . Males accounted for $40 \%$ of the study population. The mean age was $42 \pm 14$ years. The mean BMI was $29 \pm 6 \mathrm{~kg} / \mathrm{m}^{2}$. Isolated IFG, isolated IGT, combined IFG/IGT, and diabetes were present in $27 \%, 4 \%$, $9 \%$, and $21 \%$ of the participants, respectively.

Mean \pm SD age was significantly higher in individuals with isolated IFG ( $39 \pm 12$ years), isolated IGT ( $45 \pm 14$ years), and those with combined IFG/IGT ( $46 \pm 16$ years) compared with those with NGT (37 \pm 12 years). Compared with NGT, there was a significantly higher percentage of men with isolated IFG $(P<0.001)$ but a smaller percentage with isolated IGT $(P=0.03)$ and combined IFG/IGT $(P=0.007)$. BMI was significantly higher in individuals with isolated IFG $\left(29.4 \pm 5.4 \mathrm{~kg} / \mathrm{m}^{2}\right.$ vs. $\left.27.3 \pm 5.8 \mathrm{~kg} / \mathrm{m}^{2}, P=0.001\right)$ and those with combined IFG/IGT $\left(30.0 \pm 4.7 \mathrm{~kg} / \mathrm{m}^{2}\right.$ vs. $27.3 \pm 5.8 \mathrm{~kg} /$ $\left.\mathrm{m}^{2}, P=0.005\right)$ but not in those with isolated IGT $(29.1 \pm 4.9 \mathrm{~kg} /$ $\mathrm{m}^{2}$ vs. $27.3 \pm 5.8 \mathrm{~kg} / \mathrm{m}^{2}, P=0.18$ ) relative to NGT. Relative to individuals with NGT, those with combined IFG/IGT had significantly higher total cholesterol, low-density lipoprotein cholesterol, and triglyceride concentrations (all comparisons $P=0.003)$. In contrast, high-density lipoprotein cholesterol 


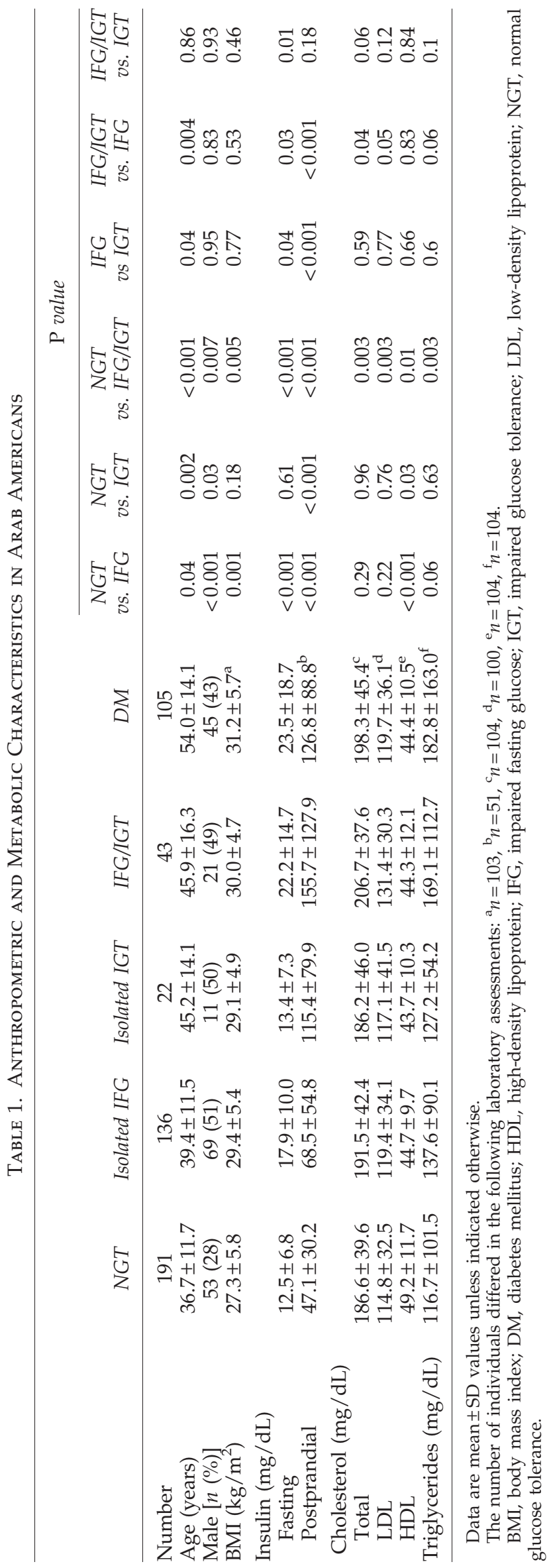

concentrations were significantly lower in those with isolated IFG, isolated IGT, and combined IFG/IGT compared with those with NGT (all comparisons $P<0.05$ ). Fasting insulin concentrations were significantly higher in individuals with isolated IFG $(P<0.001)$ and those with combined IFG/IGT $(P<0.001)$ but not in those with isolated IGT $(P=0.61)$ relative to NGT. Individuals with isolated IFG, isolated IGT, and combined IFG/IGT had significantly higher postprandial insulin concentrations compared with those with NGT (all comparisons $P<0.001)$.

As shown in Table 1, there were few differences in demographic characteristics between those with isolated IFG, isolated IGT, and combined IFG/IGT. Individuals with isolated IGT were older and had higher postprandial insulin concentrations but significantly lower fasting insulin concentrations relative to those with isolated IFG. Age, total cholesterol, and fasting and postprandial insulin concentrations were higher in individuals with combined IFG/IGT compared with those with isolated IFG. Individuals with combined IFG/IGT also had significantly higher fasting insulin concentrations relative to those with isolated IGT.

Measures of HOMA-IR, HOMA- $\% \beta$, and ISI $_{\text {composite }}$ are presented in Table 2. Compared with individuals with NGT $(2.9 \pm 1.6)$, the mean $( \pm S D)$ HOMA-IR progressively increased in individuals with isolated IFG $(4.8 \pm 2.7, P<0.001)$, combined IFG/IGT $(6.0 \pm 4.3, P<0.001)$, and diabetes $(9.7 \pm 8.3$, $P<0.001)$ but not in those with isolated IGT $(3.0 \pm 1.7, P=0.87)$. After adjustment for sex, and BMI, these associations remained unchanged. Additionally, HOMA-IR was significantly higher in individuals with isolated IFG compared with those with isolated IGT $(P=0.002)$ and with combined IFG/ IGT versus both isolated IFG $(P=0.025)$ and isolated IGT $(P=0.003)$.

Whole-body insulin sensitivity measured with the ISI $_{\text {composite }}$ was significantly lower in individuals with isolated IFG (3.9 $\pm 2.3, P<0.001)$, isolated IGT $(2.8 \pm 1.5, P<0.001)$, combined IFG/IGT $(1.9 \pm 1.1, P<0.001)$, and diabetes $(1.6 \pm 1.1$, $P<0.001)$ relative to those with NGT $(6.1 \pm 3.5)$. After adjustment for sex, and BMI, these associations remained unchanged. Furthermore, insulin sensitivity was significantly lower with isolated IGT relative to isolated IFG $(P=0.010)$ and with combined IFG/IGT versus both isolated IFG $(P<0.001)$ and isolated IGT $(P=0.013)$.

Compared with NGT $(161.3 \pm 92.0)$, HOMA- $\% \beta$ was significantly decreased with the onset of diabetes $(113.7 \pm 124.9$, $P<0.001)$. After adjustment for age, sex, and BMI, IFG was also significantly associated with a decline in $\mathrm{HOMA}-\% \beta$ relative to NGT $(P=0.005)$. HOMA- $\% \beta$ was significantly lower in individuals with isolated IFG compared with those with isolated IGT $(P=0.021)$ and combined IFG/IGT $(P=0.029)$. There were no differences noted between combined IFG/IGT versus isolated IGT $(P=0.63)$.

\section{Discussion}

This study was conducted to examine potential differences in the pathophysiologic mechanisms of insulin resistance or sensitivity and insulin secretion across the spectrum of glucose intolerance in a population-based random sample of Arab Americans. Insulin sensitivity as measured by the adjusted HOMA-IR was significantly lower with isolated IFG versus isolated IGT and in combined IFG/IGT versus isolated 


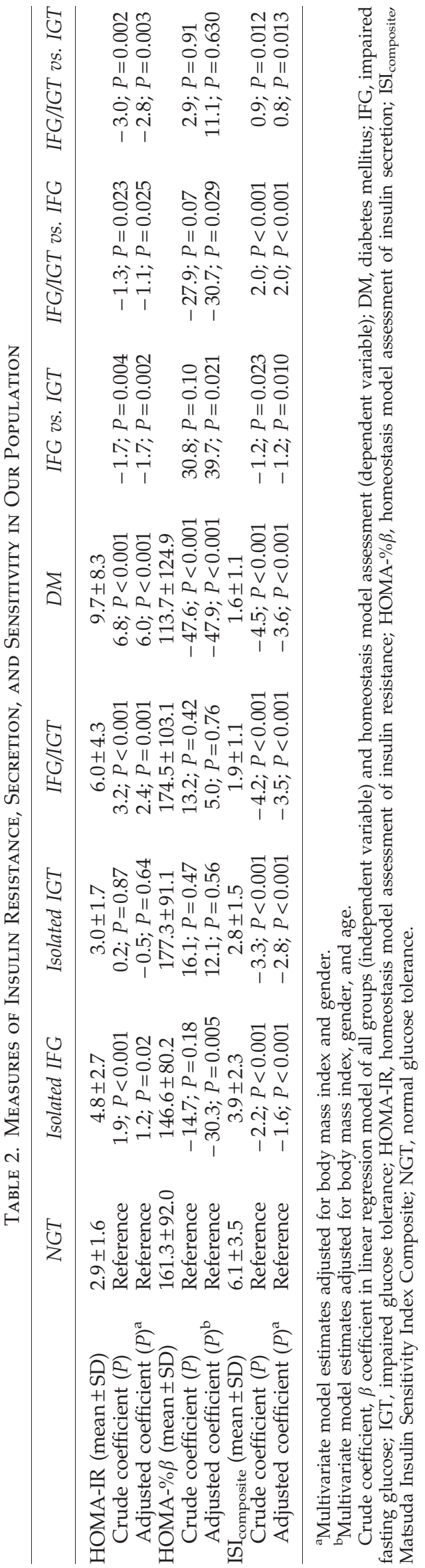

IFG and isolated IGT. On the other hand, insulin sensitivity as measured by the adjusted ISI $_{\text {composite }}$ was significantly lower with isolated IGT versus isolated IFG and in combined IFG/ IGT versus isolated IFG and isolated IGT. Adjusted HOMA$\% \beta$ was significantly lower with isolated IFG versus isolated IGT and combined IFG/IGT.

Our findings are consistent with several but not all studies that used the HOMA method for estimation of insulin resistance. Several studies have suggested that differences in HOMA-IR between the glucose intolerance states exist; insulin sensitivity is significantly decreased in individuals with isolated IFG and combined IFG/IGT. ${ }^{3,4,19-21}$ Isolated IGT using HOMA-IR was also associated with a significant decline in insulin sensitivity in some but not all studies. ${ }^{3-5,19-22}$ In addition to HOMA measurements, the insulin sensitivity index has been widely used to describe the pathophysiologic mechanisms of glucose intolerance. The ISI composite, which estimates whole-body insulin sensitivity using two sample points for plasma glucose and insulin measurements, has been shown to be comparable to insulin sensitivity measures using five sample points. ${ }^{18}$ A study of 1,264 Asian Indians has reported significant differences in NGT versus both isolated IFG and isolated IGT as calculated using HOMA-IR and significant differences in NGT versus isolated IFG, isolated IGT, combined IFG/IGT, and diabetes as calculated using the modified Matsuda index. ${ }^{23}$ Using the Matsuda ISI $_{\text {composite }}$ in our population, we have demonstrated significant differences in all categories of glucose intolerance compared with NGT and when comparing isolated IFG versus isolated IGT and combined IFG/IGT versus isolated IFG and isolated IGT. Several studies have described a significant decline in $\beta$-cell function in individuals with isolated IFG, isolated IGT, and combined IFG/IGT. ${ }^{3,4,19,20,22}$ Studies assessing insulin secretion using $\mathrm{HOMA}-\% \beta$ resulted in findings similar to those reported in this study with respect to comparisons among isolated IFG, isolated IGT, and combined IFG/IGT. ${ }^{5,20,22}$ There are several potential limitations to our study. First, we used the HOMA method for estimating insulin resistance and secretion in this population-based randomly selected sample of Arab Americans. Although this method has been correlated to the hyperinsulinemic-euglycemic clamp, fasting glucose levels significantly influence the accuracy of HOMA estimation indices. ${ }^{4,24}$ In addition, the indices in the formula do not account for potential variations in insulin clearance. Second, postprandial insulin concentrations were derived from a single OGTT testing, limiting our ability to assess the hyperbolic relation between insulin secretion to insulin sensitivity in individuals with isolated IFG, isolated IGT, and combined IFG/IGT. ${ }^{25}$ Third, IFG was the most prevalent abnormality followed by diabetes in our population. Using the current American Diabetes Association criteria, the prevalence of IFG of $27 \%$ shown here is $19 \%$ higher than the rate we previously reported, stemming from a disproportionately high number of individuals with isolated IFG compared with those with isolated IGT or combined IFG/IGT. ${ }^{12,16}$ It is plausible that the lack of significant changes in HOMA- $\% \beta$ shown here in individuals with isolated IGT or combined IFG/IGT may in part be due to the small sample size of these groups. Fourth, there are multiple factors that can influence insulin resistance such as waist-hip ratio, hypertension, hypertriglyceridemia, low high-density lipoprotein cholesterol, C-peptide, hemoglobin A1C, and fasting insulinemia that could not be systematically 
assessed. Finally, the cross-sectional design of our study limited the ability to assess the natural progression of these metabolic abnormalities across the disease continuum.

In conclusion, given the high prevalence of prediabetes and diabetes in the Arab American population, a clear understanding of the underlying pathophysiological mechanisms of the glucose intolerance states leading to diabetes is important. This study suggests that differences in the metabolic defects in Arab Americans with IFG and/or IGT may exist and may require different strategies for the prevention of diabetes. It appears that interventions that encompass enhancing insulin sensitivity and preserving $\beta$-cell function may be more efficacious for preventing diabetes in this vulnerable population.

\section{Acknowledgments}

This study was funded by the American Diabetes Association. F.D.S. contributed to the discussion and wrote the manuscript. N.R.P. contributed to the discussion and wrote the manuscript. E.T.M. contributed to the discussion and reviewed/edited the manuscript. L.A.J. researched data, contributed to the discussion, and reviewed/edited the manuscript.

\section{Author Disclosure Statement}

No competing financial interests exist.

\section{References}

1. Lillioja S, Mott DM, Spraul M, Ferraro R, Foley JE, Ravussin E, Knowler WC, Bennett PH, Bogardus C: Insulin resistance and insulin secretory dysfunction as precursors of noninsulin-dependent diabetes mellitus. Prospective studies of Pima Indians. N Engl J Med 1993;329:1988-1992.

2. Haffner SM, Miettinen H, Gaskill SP, Stern MP: Decreased insulin secretion and increased insulin resistance are independently related to the 7-year risk of NIDDM in MexicanAmericans. Diabetes 1995;44:1386-1391.

3. Faerch K, Vaag A, Holst JJ, Hansen T, Jørgensen T, BorchJohnsen K: Natural history of insulin sensitivity and insulin secretion in the progression from normal glucose tolerance to impaired fasting glycemia and impaired glucose tolerance: the Inter99 study. Diabetes Care 2009;32:439-444.

4. Abdul-Ghani MA, Jenkinson CP, Richardson DK, Tripathy D, DeFronzo RA: Insulin secretion and action in subjects with impaired fasting glucose and impaired glucose tolerance: results from the Veterans Administration Genetic Epidemiology Study. Diabetes 2006;55:1430-1435.

5. Meyer C, Pimenta W, Woerle HJ, Van Haeften T, Szoke E, Mitrakou A, Gerich J: Different mechanisms for impaired fasting glucose and impaired postprandial glucose tolerance in humans. Diabetes Care 2006;29:1909-1914.

6. Weyer C, Bogardus C, Pratley RE: Metabolic characteristics of individuals with impaired fasting glucose and/or impaired glucose tolerance. Diabetes 1999;48:2197-2203.

7. Faerch K, Vaag A, Holst JJ, Glümer C, Pedersen O, BorchJohnsen K: Impaired fasting glycaemia vs impaired glucose tolerance: similar impairment of pancreatic alpha and beta cell function but differential roles of incretin hormones and insulin action. Diabetologia 2008;51:853-861.

8. Festa A, D'Agostino R Jr, Hanley AJ, Karter AJ, Saad MF, Haffner SM: Differences in insulin resistance in nondiabetic subjects with isolated impaired glucose tolerance or isolated impaired fasting glucose. Diabetes 2004;53:1549-1555.

9. Abdul-Ghani MA, Tripathy D, DeFronzo RA: Contributions of beta-cell dysfunction and insulin resistance to the pathogenesis of impaired glucose tolerance and impaired fasting glucose. Diabetes Care 2006;29:1130-1139.

10. Abdul-Ghani MA, Matsuda M, Sabbah M, Jenkinson CP, Richardson DK, Kaku K, DeFronzo RA: The relative contributions of insulin resistance and beta cell failure to the transition from normal to impaired glucose tolerance varies in different ethnic groups. Diabetes Metab Syndr Clin Res $\underline{\operatorname{Rev}}$ 2007;1:105-112.

11. Shaat N, Ekelund M, Lernmark A, Ivarsson S, Nilsson A, Perfekt R, Berntorp K, Groop L: Genotypic and phenotypic differences between Arabian and Scandinavian women with gestational diabetes mellitus. Diabetologia 2004;47: 878-884.

12. Jaber LA, Brown MB, Hammad A, Nowak SN, Zhu Q, Ghafoor A, Herman WH: Epidemiology of diabetes among Arab Americans. Diabetes Care 2003;26:308-313.

13. DeFronzo RA, Abdul-Ghani MA: Type 2 diabetes can be prevented with early pharmacological intervention. Diabetes Care 2011;34(Suppl 2):S202-S209.

14. Salinitri FD, Martin ET, Pinelli NR, Jaber LA: Insulin resistance and secretion in Arab Americans with glucose intolerance [abstract 1488-P]. Diabetes 2012;61(Suppl 1):A387.

15. Friedewald WT, Levy RI, Fredrickson DS: Estimation of the concentration of low-density lipoprotein cholesterol in plasma, without use of the preparative ultracentrifuge. Clin Chem 1972;18:499-502.

16. American Diabetes Association: Diagnosis and classification of diabetes mellitus. Diabetes Care 2012;35(Suppl 1):S64-S71.

17. Matthews DR, Hosker JP, Rudenski AS, Naylor BA, Treacher DF, Turner RC: Homeostasis model assessment: insulin resistance and beta-cell function from fasting plasma glucose and insulin concentrations in man. Diabetologia 1985;28: 412-419.

18. DeFronzo RA, Matsuda M: Reduced time points to calculate the composite index. Diabetes Care 2010;33:e93.

19. Hanefeld M, Koehler C, Fuecker K, Henkel E, Schaper F, Temelkova-Kurktschiev $\mathrm{T}$ : Insulin secretion and insulin sensitivity pattern is different in isolated impaired glucose tolerance and impaired fasting glucose: the risk factor in Impaired Glucose Tolerance for Atherosclerosis and Diabetes study. Diabetes Care 2003;26:868-874.

12. Snehalatha C, Ramachandran A, Sivasankari S, Satyavani $\mathrm{K}$, Vijay V: Insulin secretion and action show differences in impaired fasting glucose and in impaired glucose tolerance in Asian Indians. Diabetes Metab Res Rev 2003;19: 329-332.

21. Tripathy D, Almgren P, Tuomi T, Groop L: Contribution of insulin-stimulated glucose uptake and basal hepatic insulin sensitivity to surrogate measures of insulin sensitivity. Diabetes Care 2004;27:2204-2210.

22. Davies MJ, Raymond NT, Day JL, Hales CN, Burden AC: Impaired glucose tolerance and fasting hyperglycaemia have different characteristics. Diabet Med 2000;17:433-440.

23. Staimez LA, Weber MB, Ranjani H, Ali MK, EchouffoTcheugui JB, Phillips LS, Mohan V, Narayan KM: Evidence of reduced beta cell function in Asian Indians with mild dysglycemia. Diabetes Care 2013 April 17 [Epub ahead of print]. doi: 10.2337/dc12-2290. 
24. Bonora E, Targher G, Alberiche M, Bonadonna RC, Saggiani F, Zenere MB, Monauni T, Muggeo M: Homeostasis model assessment closely mirrors the glucose clamp technique in the assessment of insulin sensitivity: studies in subjects with various degrees of glucose tolerance and insulin sensitivity. Diabetes Care 2000;23:57-63.

25. Retnakaran R, Shen S, Hanley AJ, Vuksan V, Hamilton JK, Zinman B: Hyperbolic relationship between insulin secretion and sensitivity on oral glucose tolerance test. Obesity (Silver Spring) 2008;16:1901-1907.
Address correspondence to:

Francine D. Salinitri, PharmD

Department of Pharmacy Practice Wayne State University Eugene Applebaum College of Pharmacy and Health Sciences

259 Mack Avenue, Suite 2190

Detroit, MI 48201

E-mail: fsalinitri@wayne.edu 
This article has been cited by:

1. Kyle J Burghardt, Dana El Masri, Sabrina E Dass, Sara S Shikwana, Linda A Jaber. 2017. Association between haptoglobin gene and insulin resistance in Arab-Americans. Biomarkers in Medicine 11:11, 937-945. [Crossref] 